\title{
IR heat transfer measurements on a rotating disk
}

\author{
by CARDONE G., ASTARITA T. and CARLOMAGNO G.M.
}

\author{
University of Naples, DETEC, P.le Tecchio 80, 80125 Naples, Italy
}

\section{Abstract}

Heat transfer measurements on a rotating disk are performed, for a wide range of Reynolds number values in the laminar, transitional and turbulent regimes, by making use of the heated-thin-foil technique and by measuring temperature maps with an infrared scanning radiometer. The use of the IR radiometer is advantageous on account of its relatively good spatial resolution and thermal sensitivity and because it allows one to perform measurements down to very low Reynolds numbers. Heat transfer results are presented in terms of Nusselt and Reynolds numbers based on the local radius and show a substantial agreement with previous experimental and theoretical analyses. Transition to turbulent flow is found at about $R e=250,000$. A discussion about the role played by the adiabatic wall temperature is included.

\section{Introduction}

The laminar flow due to an infinite flat disk rotating in still air is one of the few exact solutions to the three-dimensional Navier-Stokes equations. This type of flow was first theoretically investigated with an approximate method by von Kàrmàn (1921) [1] who found that it resembles a boundary layer flow but with a boundary layer thickness independent of the radial distance. The tangential component of the shear stress at the disk surface imparts a circunferential velocity to the adjacent fluid layer which in turn, due to the centrifugal forces, moves also radially outwards. Rogers and Lance (1960) [2] calculated accurate solutions by means of a numerical integration of the governing equations.

Wagner (1948) [3] first evaluated the convective heat transfer coefficient by finding an approximate solution in the laminar regime based on the von Kàrmàn velocity distribution. The Wagner relation between the local Nusselt and Reynolds numbers is:

$$
\mathrm{Nu}=\mathrm{a} \sqrt{\operatorname{Re}}
$$

where $a$ is a constant which is equal to 0.335 for $\operatorname{Pr}=0.74$. The local Nusselt and Reynolds numbers are respectively defined as:

$$
N u=\frac{h r}{k} \quad R e=\frac{\omega r^{2}}{v}
$$

where $\boldsymbol{h}$ is the local convective heat transfer coefficient, $r$ is the local radius, $\omega$ is the angular speed, $k$ and $v$ are the fluid thermal conductivity and kinematic viscosity coefficients, respectively.

It should be explicitly evidenced that eq. (1) assumes $h$ to be constant over the whole surface of the disk so that both constant temperature and heat flux boundary conditions apply. Millsaps and Pohlhausen (1952) [4] solved, always in the laminar regime, the exact equation of the thermal field by including viscous dissipation effects and find the coefficient a to be equal to 0.334 when viscous dissipation is neglected and $\operatorname{Pr}=0.74$.

Cobb and Saunders (1956) [5] performed an experimental investigation on the mean heat transfer coefficient for a range of conditions from entirely laminar to mixed laminar 
http://dx.doi.org/10.21611/qirt.1994.024

turbulent flow. In the laminar regime, they pointed out that eq. (1) is still valid (with the same value of the constant a) provided the average $\mathrm{Nu}$ and $\mathrm{Re}$ numbers are used. The lowest tested Reynolds number based on the disk radius is about 100,000 . Although their data show a dependence of $\mathrm{Nu}$ from $\mathrm{Re}$ with an exponent which seems lower than 0.5 , they affirmed that, in the laminar range, experimental results fit eq. (1) with a coefficient a equal to 0.36 . Moreover at the lowest tested $\mathrm{Re}$, their results are much higher than what predicted by eq. (1). This is due to the influence of the natural convection around the disk which becomes important at the low rotational speeds they tested. Cobb and Saunders detected the onset of transition to turbulent flow at about $R e=240,000$. They yielded also the following correlation between the local Nusselt and Reynolds numbers in the turbulent regime:

$$
N u=0.0193 R e^{0.8}
$$

which was obtained by means of the classic Reynolds analogy and experimental data of the turbulent mean friction moment coefficient of Theodorsen and Regier (1944) [6].

Kreith et al. (1959) [7] experimentally evaluated mass transfer rates from a rotating disk of naphthalene under laminar and turbulent conditions and related their results to heat transfer coefficients by means of an analogy method. They found a good agreement with theoretical data in the laminar range and located the onset of transition in the range $200,000<\operatorname{Re}<$ 250,000 .

Popiel and Boguslawski (1975) [8] measured the local heat transfer coefficient at a certain location over a disk rotating at different angular speeds and found the coefficient a to be equal to 0.33 for laminar conditions. Nu seems to depend on Re with an exponent much lower than 0.5 from their results too. In fact, at the lowest tested Reynolds number $(\approx 7000)$, Popiel and Boguslawski, who took into account also the effects of natural convection, measured a Nusselt number which is about 35\% larger than the theoretical predictions. They found the onset of transition at about $R e=195,000$. In the transitional and turbulent regimes their experimental data fit respectively the relationships:

$$
N u=10^{-19} R e^{4}
$$

for $R$ e ranging from 195,000 to 250,000 and:

$$
\mathrm{Nu}=0.0188 \operatorname{Re}^{0.8}
$$

for Re greater than 250,000 .

The aim of this paper is to discuss critically the validity limits of the previous correlations of literature, in particular as far as the transitional flow regime is concerned. A major peculiarity of the present work lies in the fact that an infrared (IR) imaging system (IR scanning radiometer or thermograph) is employed to measure the disk surface temperature, and so the wall heat transfer coefficient. The advantage of having a non-contact temperature measuring device, such as the IR scanning radiometer, is well exploited to carry out heat transfer measurements in the vicinity of the disk axis of rotation, i.e. down to very low local Reynolds numbers.

\section{Experimental apparatus and procedure}

The sketch of the experimental apparatus is shown in Fig 1. The disk section consists of a $300 \mathrm{~mm}$ (or $450 \mathrm{~mm}$ ) in diameter aluminium (or light-alloy steel) cup filled with a $20 \mathrm{~mm}$ thick polyurethane foam on which a printed circuit board is glued. The circuit is used to generate, by Joule effect, an uniform heat flux on the disk surface, while the polyurethane foam thermally insulates the face of the disk not exposed to air. Electric power is supplied to the printed circuit by means of a mercury rotating contact. Details about printed circuit are reported in Ref [9]

A pulley, which is connected by a transmission belt to an electric motor, is fixed on the transmission shaft supporting the disk. The rotating speed of the disk, which may be positioned either normal or parallel to the gravitational force, can be varied in a continuos way 
http://dx.doi.org/10.21611/qirt.1994.024

within the range $100-4500 \mathrm{~mm}$ by changing the motor pulley and/or by means of an inverter. The disk angular speed is continuously monitored by a tachometer.

To enhance the IR radiation detection, the measured board surface is coated with a thin layer of black paint which has a total emissivity coefficient equal to 0.95 in the working IR window of the employed IR scanner.

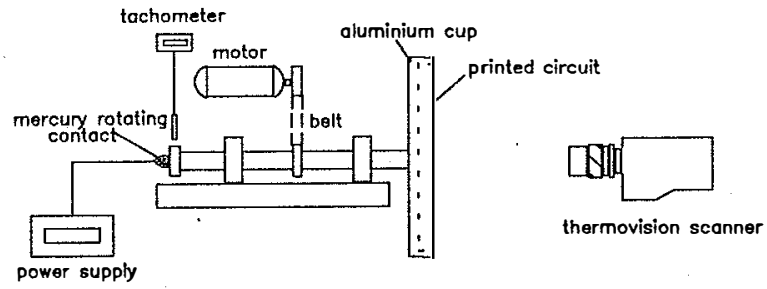

Fig. 1 - Experimental apparatus.

The infrared thermographic system is based on AGEMA Thermovision 880 . The field of view (which depends on the optic focal length and on the viewing distance) is scanned by the $\mathrm{Hg}$-Cd-Te detector in the $8-12 \mu \mathrm{m}$ IR window. Nominal sensitivity, expressed in terms of noise equivalent temperature difference is $0.1^{\circ} \mathrm{C}$ when the scanned object is at ambient temperature. The scanning spatial resolution is 175 instantaneous fields of view per line at $50 \%$ slit response function. A $20^{\circ} \times 20^{\circ}$. lens is used during the tests. The thermal image is digitized in a frame of $140 \times 140$ pixels $\times 8$ bits.

An application software is developed to correlate measured temperatures to heat transfer coefficients by means of the so called heated-thin-foil technique [10]:

$$
h=\frac{q_{J}-q_{r}}{T_{w}-T_{a w}}
$$

where $q_{j}$ is the Joule heating, $q_{r}$ is the radiative heat flux to ambient, $T_{w}$ is the measured wall temperature and $T_{a w}$ the adiabatic wall temperature of the flow. The radiative thermal losses $q_{r}$ are computed by using the measured $T_{w}$, while the conductive ones toward the inner polyurethane foam, are neglected. $T_{a w}$ is measured by means of the thermographic technique under the assumption that it coincides with the disk surface temperature when the Joule heating is suppressed. The role played by the use of $T_{a w}$ in eq. (6) in place of the ambient temperature $T_{a}$ will be discussed later.

\section{Results}

In Fig. 2 a thermographic image of the disk, $(D=300 \mathrm{~mm})$ rotating at $576 \mathrm{rm}$ and subject to a heat flux $q_{J}=407 \mathrm{~W} / \mathrm{m}^{2}$, is shown. As expected for laminar regime, most of the disk surface appears at constant temperature because of the constant imposed heat flux and of the constant convective and radiative heat transfer coefficients.

Fig. 3 is a thermal picture of the disk $(D=450 \mathrm{~mm})$ recorded while it is rotating at $4390 \mathrm{~mm}$ and is subject to a heat flux of $871 \mathrm{WI} \mathrm{m}^{2}$. A relatively small (about $16 \%$ of the total surface) region around the disk center, where temperature is constant, is clearly evident. On the basis of the previous discussion the flow is laminar there. In the outer zon'e the temperature decreases, first quickly in the transitional regime and then slowly in the turbulent one; near the disk edge the temperature trend is reversed as it begins to rise slowly.

In order to explain the temperature behavior in the turbulent regime, first consider that due to the turbulent correlation law (e.g. eq. (3)) the heat transfer coefficient is expected to 
http://dx.doi.org/10.21611/qirt.1994.024

increase as the local radius increases. As long as the adiabatic wall temperature distribution is uniform eq. (6) shows that the wall temperature must decrease along the radial direction. Indeed, by examining the cold thermogram of Fig. 4 , relative to the adiabatic wall temperature recorded at the same disk angular speed as Fig. 3 , it should be noted that $T_{a w}$ is practically constant (and equal to $T_{a}$ ) within the circumference whose radius is about $60 \%$ of the disk one.

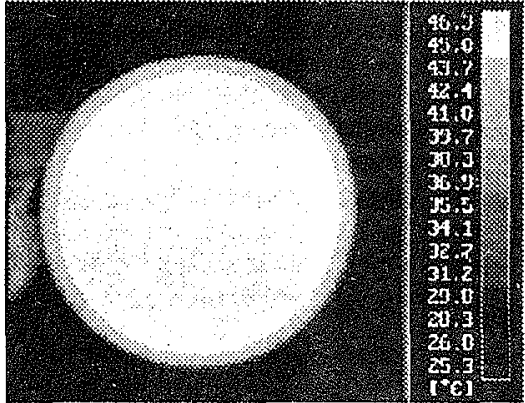

Fig. 2 - Thermogram for $\omega=576 \mathrm{rpm}$ and $q_{j}=487 \mathrm{~W} / \mathrm{m}^{2}$

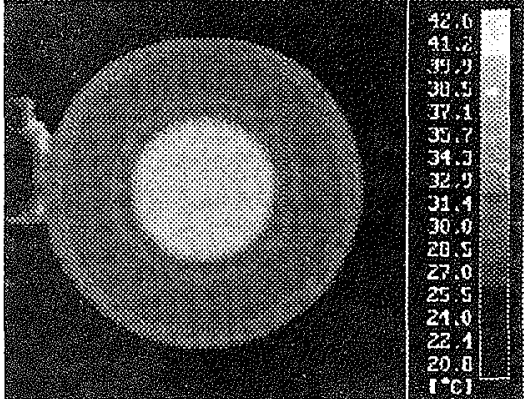

Fig. 3 - Thermogram for $\omega=4390 \mathrm{rpm}$ and $q_{j}=871 \mathrm{~W} / \mathrm{m}^{2}$

The $T_{w}$ trend in this region is thus confirmed. Close to the disk limb $T_{a w}$ experiences a significant increase (about $3^{\circ} \mathrm{C}$ over $T_{a}$ at the disk edge); for the present experimental conditions ( $T_{w}$ is of the same order of magnitude as $T_{a w}$ ) the increasing trend of the wall temperature (Fig. 3 ) is accordingly recovered. It has to be explicitly pointed out that in the case of relatively high boundary heat fluxes $q_{j}$ or high $\Delta T=T_{w}-T_{a w}$ temperatures differences, the effect of the adiabatic wall temperature is negligible and the expected $T_{w}$ trend is monotonically decreasing.

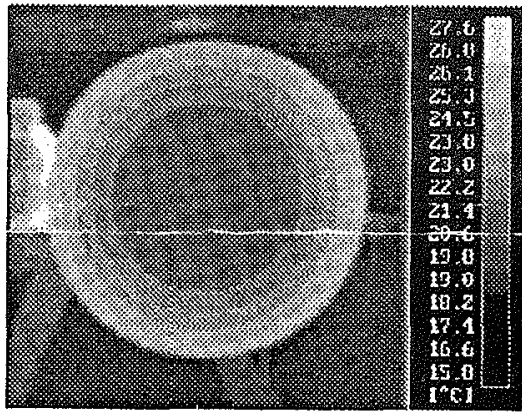

Fig. 4- Thermogram for $\omega=4390 \mathrm{rpm}$ and $q_{j}=0 \mathrm{~W} / \mathrm{m}^{2}$

Results of performed tests, with both the $300 \mathrm{~mm}$ and $450 \mathrm{~mm}$ in diameter disks are shown in Fig. 5 in terms of local $\mathrm{Nu}$ and Re numbers where both $k$ and $v$ are evaluated at film temperature. The theoretical prediction of Millsaps and Pohlhausen is also reported. The almost sudden rise of $\mathrm{Nu}$ around $\mathrm{Re}=250,000$ is to be attributed to the onset of transition from laminar to turbulent flow. In the laminar zone, all the points fall around a straight line in the log-log plane down to very low Reynolds numbers. Following the Wagner theory, a correlation of all the data in terms of equation (1) is made and the value of the constant is 
http://dx.doi.org/10.21611/qirt.1994.024

found to be $a=0.33$. This value looks in very good agreement with previous both theoretical and experimental findings. It has to be stressed that the validity of (1) down to very low local Reynolds numbers has been proved.

To check the actual slope of data in the log-log plane, a generalization of Wagner relation is investigated, namely:

$$
N u=a^{\prime} e^{b}
$$

A linear regression based on eq. (7) is applied to a limited sample of data, first by neglecting $T_{a w}$ effects and then by taking them into account. In the former case the evaluated slope is $b=0.488$, in the latter one $b=0.499$. This finding should demonstrate that the not very accurate correlation of previous investigators is to be attributed to the $T_{a w}$ effect which was systematically neglected.

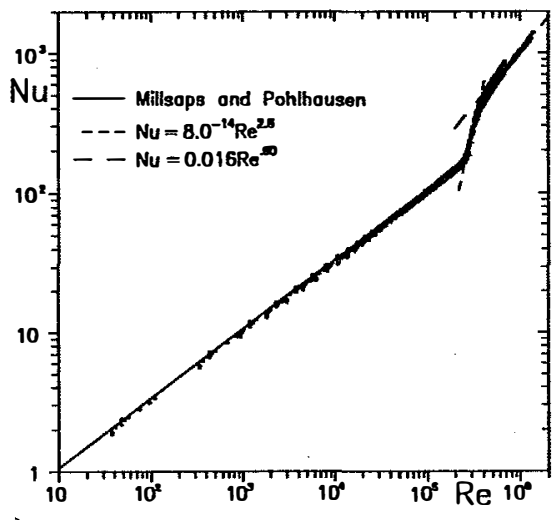

Fig. 5 - Nusselt number against Reynolds number.

As said before the almost sudden rise of $\mathrm{Nu}$ around $\mathrm{Re}=250,000$ is to be attributed to the onset of transition while the second slope change, which appears at the right end of figure, may be attributed to the presence of fully turbulent flow. A linear regression of the data in the transitional range of $R e$ from 260,000 to 320,000 yields:

$$
N u=8.010^{-14} \operatorname{Re}^{2.8}
$$

Present transitional results do not agree at all with those of Ref. [8] as far as both the transitional $R e$ range and the regression slope are concerned. Apart from the remark that measurements in transitional flow regimes may be in general strongly affected by the environmental conditions of the actual experimental apparatus, it should be stressed that the results of Ref. [8] are obtained by using a calorimetric device too large to achieve the fine spatial resolution exhibited by the data reported in the cited paper.

In the fully turbulent regime present data fit the relation:

$$
N u=0.016 R e^{0.8}
$$

which is in good agreement with the theoretical and experimental findings of the literature.

It has to be finally remarked that the obtained results are practically identical for either disk normal to the direction of the gravitational force or disk parallel to this latter and facing downward; this behavior confirms the neglecting the natural convection effects, for the tested rotational speeds, to be rightful. 
http://dx.doi.org/10.21611/qirt.1994.024

\section{Conclusions}

Heat transfer measurements on a rotating disk are performed in laminar, transitional and turbulent regime by making use of the heated-thin-foil technique and by measuring temperature maps with an infrared scanning radiometer. The use of the radiometer is proved to be advantageous on account of its relatively good spatial resolution and thermal sensitivity and because it allows to easily make measurements down to very low local Reynolds numbers.

Experimental data are correlated in terms of $\mathrm{Nu}$ and $\mathrm{Re}$ numbers both based on the local radius and well agree with theoretical predictions for a range of Reynolds numbers much wider than that obtained in previous works. Moreover, in the authors opinion, transitional results seem to be more reliable than the ones available in the literature. In particular, the onset of the transition to turbulence is found around $R e=250,000$.

A discussion about the role played by the adiabatic wall temperature is also made. The experimental evidence of a $T_{a w}$ rise towards the disk limb gives an explanation of the (perhaps not expected) increase of the heated disk surface temperature in that region.

\section{REFERENCES}

[1] VON KÀRMÀN (TH.): Laminare und Turbulente Reibung, vol 1, ZAMM, pp. 233-252, 1921.

[2] ROGERS (M. G.) and LANCE (G.N.): The Rotationally Symmetric Flow of a Viscous Fluid in the Presence of an Infinite Rotating Disk, vol. 7, J. Fluid Mech., pp. 617-631., 1960.

[3] WAGNER (C.): Heat Transfer from a Rotating Disk to Ambient Air, vol. 19, J. Applied Physics, pp 837-839, 1948.

[4] MILLSAPS (K.) and POHLHAUSEN (K.): Heat Transfer by Laminar Flow from a Rotating Plate, vol. 19, J. Aeronautical Science, pp. 120-126, 1952.

[5] COBB (E. C.) and SAUNDERS (O. A.): Heat Transfer from a Rotating Disk, vol. 236, Proc. Royal Society, pp 343-351, 1956.

[6] THEODERSEN (T.) and REGIER (A.): Experiments on Drag of Revolving Disk, Cylinders, and Streamline Rods at High Speed, N.A.C.A. Tech. Rep. $n^{\circ} 793,1944$.

[7] KREITH (F.), TAYLOR (J. H.) and CHONG (J. P.): Heat and Mass Transfer From a Rotating Disk, J. vol. 81, Heat Transfer, pp. 95-105, 1959.

[8] POPIEL (CZ. O.) and BOGUSLAWSKI (L.): Local Heat-Transfer Coefficients on the Rotating Disk in Still Air, Vol. 18, Int. J. Heat Mass Transfer, pp. 167-170, 1975.

[9] CARDONE (G.), ASTARITA (T.) and CARLOMAGNO (G.M.): Infrared Themography to Measure Local Heat Transfer Coefficients on a Disk Rotating in Still Air, Proc. Workshop on Advanced Infrared Technology and Applications, pp. 143-153 1993.

[10] CARLOMAGNO (G. M.) and DE LUCA (L.): Infrared Thermography in Heat Transfer, in Handbook of Flow Visualization, Ch. 32, pp. 531-553, Hemisphere, 1989. 